COMMENTARY

\title{
How could we improve the increased cardiovascular mortality in patients with overt and subclinical hyperthyroidism?
}

\author{
Bernadette Biondi \\ Department of Clinical and Molecular Endocrinology and Oncology, University of Naples Federico II, Via S. Pansini 5, 80131 Naples, Italy \\ (Correspondence should be addressed to B Biondi; Email: bebiondi@unina.it)
}

\begin{abstract}
Over the past five years several meta-analyses have evaluated the cardiovascular mortality in patients with hyperthyroidism. They assessed various studies in which different inclusion criteria were used for the analysis of the cardiovascular mortality. More selective criteria have been used in recent metaanalyses. Only prospective cohort studies were included and only cohorts using second and third generation TSH assays were chosen. In addition, only the studies where the TSH evaluation was repeated during the follow-up were selected. The results of these recent meta-analyses provide evidence that overt and subclinical hyperthyroidism, particularly in patients with undetectable serum TSH, may increase the cardiovascular mortality. However, still today, the results remain inconclusive and not sufficient enough to recommend treatment for patients with low-detectable serum TSH. The high cardiovascular risk and mortality in presence of thyroid hormone excess suggest that this dysfunction is an important health problem and requires guidelines for the treatment of patients at high cardiovascular risk. Rigorous studies are necessary to evaluate the effects of the various causes of hyperthyroidism on the clinical outcomes. Randomized controlled clinical trials are needed to assess the benefits of treatment to improve the cardiovascular mortality and morbidity of mild and overt hyperthyroidism.
\end{abstract}

European Journal of Endocrinology 167 295-299

\section{Introduction}

Parry (1) and Graves (2) reported the first descriptions of the cardiovascular (CV) manifestations of hyperthyroidism in 1800. However, only in the last 5 years have several meta-analyses recognized that hyperthyroidism, in both overt and subclinical conditions, is associated with an increased CV mortality (3, 4, 5, 6, 7, 8, 9). Three of these meta-analyses have been published in the European Journal of Endocrinology $(4,7,8)$.

\section{CV mortality in overt hyperthyroidism}

Only two meta-analyses have assessed the CV mortality in overt hyperthyroidism $(3,4)$ (Table 1 ). The first metaanalysis was published in 2007 and evaluated the results of seven cohort studies (3); a 1.7-fold elevated risk for $\mathrm{CV}$ mortality rate was detected in the presence of overt thyroid hormone excess (3). In particular, an increased circulatory mortality was observed in three studies $(10,11,12)$.

A critical review and meta-analysis published in 2011 reported a 20\% increased mortality in patients with overt hyperthyroidism and six studies assessing CV mortality were included (4). When the authors pooled them together, they were associated with a higher $\mathrm{CV}$ mortality in overt hyperthyroidism compared with the control population (relative risk (RR) 1.13; 95\% confidence interval (CI) 1.00 to 1.28) (4). Particularly, in this last meta-analysis, four studies $(10,13,14,15)$ found a significantly raised CV mortality in hyperthyroid patients compared with the control population. The majority of epidemiological studies assessing CV mortality in overt hyperthyroidism only adjusted their results for age, sex, and race as relevant confounders; moreover, in some studies, the gender-matched control group was inhomogeneous $(3,4)$.

Some of the studies included in these two metaanalyses were performed in Europe where iodine deficiency determines the increased risk of hyperthyroidism due to thyroid autonomy (toxic multinodular goiter (TMNG) and toxic adenoma (TA)) (16). Other studies came from the United States, where Graves disease (GD) is the main form of hyperthyroidism because iodine sufficiency is present in this country (16).

It is important to identify the potential cardiac events responsible for the increased $\mathrm{CV}$ mortality in the presence of thyroid hormone excess. The etiology of hyperthyroidism, the severity, duration, and age of this dysfunction, the association with smoking, genetic, and environmental factors, underlying heart conditions, or comorbidities may influence the CV risk $(16,17)$. In the majority of the epidemiological studies, these 
Table 1 Summary of meta-analyses assessing the cardiovascular mortality in the presence of thyroid hormone excess.

\begin{tabular}{lc}
\hline References & Studies included \\
\hline $\begin{array}{l}\text { Meta-analyses on cardiovascular mortality } \\
\text { in patients with overt hyperthyroidism }\end{array}$ \\
$\quad$ Völzke et al. (3) & 7 \\
Brandt et al. (4) & 6 \\
Meta-analyses on cardiovascular mortality in & \\
patients with subclinical hyperthyroidism & \\
Völzke et al. (1) & 2 \\
Singh et al. (5) & 3 \\
Ochs et al. (6) & 5 \\
Haentjens et al. (7) & 7 \\
Yang et al. (8) & 17 \\
Collet et al. (9) & 10 \\
\hline
\end{tabular}

confounders were not considered, and this could have severely limited the recognition of the potential causes responsible for the increased CV mortality in overt hyperthyroidism.

Thyroid autonomy usually develops in elderly patients who may have underlying heart disease or comorbidity and it is preceded by prolonged subclinical hyperthyroidism (SHyper) (16, 17). Persistent atrial fibrillation with left atrial enlargement, embolic events, systolic hypertension with a wide pulse pressure, heart failure (HF), and coronary heart disease (CHD) represent the most frequent adverse cardiac events observed in elderly patients with hyperthyroidism, especially in patients with associated heart disease $(16,17)$. GD usually develops in young and middle-aged patients with severe hyperthyroidism; moreover, it may be associated with autoimmune $\mathrm{CV}$ involvement with the potential onset of pulmonary arterial hypertension, cardiac valve involvement, and dilated cardiomyopathy (16). Therefore, different $\mathrm{CV}$ risk factors might be associated with different etiologies of hyperthyroidism, although this assumption remains to be demonstrated after the evaluation of large clinical studies, comparing the CV risk in patients with different causes of hyperthyroidism.

\section{$\mathrm{CV}$ risk during treatment with antithyroid drugs}

It is essential to improve the $\mathrm{CV}$ mortality with an appropriate treatment of overt hyperthyroidism. The persistence of some $\mathrm{CV}$ risk factors has been reported in some studies during the treatment of overt thyroid dysfunction $(13,18)$. Supraventricular dysrhythmias, CV symptoms, and negative hemodynamic changes persisted after treatment with antithyroid drugs in the prospective study performed by Osman et al. (13). These data suggest a delay in restoring the tissue euthyroidism during medical therapy of hyperthyroidism, despite the normalization of thyroid hormone levels. In fact, TSH levels may be persistently low to undetectable during treatment with antithyroid drugs and do not allow restoration of sinus rhythm in patients with atrial fibrillation and improvement of cardiac function in the presence of heart disease $(19,20)$.

Atrial fibrillation is an important cause of stroke and pulmonary embolism $(21,22)$ and may be reversible after treatment of hyperthyroidism (23). Spontaneous reversion occurs in up to $50 \%$ of adequately treated patients (23). However, the reversion of atrial fibrillation rarely happens in patients with advanced age, history of cardiac failure, and the presence of associated $\mathrm{CV}$ risk factors such as diabetes, left ventricular hypertrophy, elevated systolic and/or diastolic blood pressure, and atrial enlargement $(13,23)$. These considerations could suggest the importance of rapidly normalizing serum TSH levels during treatment with antithyroid drugs in elderly patients and in those with associated CV risk factors. Moreover, special attention should be given to an adequate and prompt treatment of atrial fibrillation to avoid its persistence, mainly in patients with new onset atrial fibrillation with the appearance of hyperthyroidism.

\section{CV mortality after radioiodine treatment}

An increased mortality, although statistically nonsignificant, was reported in the meta-analysis by Brandt et al. (4) after pooling all studies assessing the risk of hyperthyroidism after radio active iodine (RAI) treatment. An overall increased all cause mortality and CV mortality was reported in four studies after radioiodine treatment $(14,15,24,25)$. Interestingly, the majority of these studies adjusted their analyses for sex, age, $\mathrm{CV}$ disease (CVD), and comorbidities.

Total CVD and fractures were the major significant factors contributing to the increased mortality in the study by Franklyn et al. (15). The excess mortality was associated with cerebrovascular disease and ischemic heart disease, especially in subjects older than 60 years (15).

Some studies differentiated the $\mathrm{CV}$ risk after RAI in patients with GD and TMNG $(14,25)$. An increased rate of hospitalization for arrhythmias, atrial fibrillation, cerebrovascular disease, and HF was reported after RAI, particularly in patients with TMNG $(14,25)$. Cerebrovascular diseases were the most important causes of mortality in patients with TMNG aged over 65 years, whereas RAI was not associated with an increased CV mortality in GD (14).

It is not clear whether the increased CV mortality after radioiodine is due to the severity of hyperthyroidism itself or to the negative effects in hyperthyroid patients inadequately prepared for RAI. In some studies, a strong link was observed between mortality and the dose of radioiodine as an indirect marker for the severity of hyperthyroidism. RAI may lead to a worsening of hyperthyroidism and of its CV implications because it induces thyroiditis due to radiation. This effect is 
particularly dangerous in older patients and in subjects with severe hyperthyroidism. McDermott et al. (26) reported some cases of thyroid storm after RAI.

The recent American Thyroid Association guidelines on the treatment of hyperthyroidism have suggested that treatment with beta-blocking drugs may be sufficient to control severe hyperthyroidism before RAI. However, they have reported conflicting opinions on pretreatment with antithyroid drugs (Recommendation 33) (27). Previous considerations could suggest that hyperthyroidism should be treated before RAI especially in severe disease, elderly patients, and in those with atrial fibrillation (AF) and CVD.

A meta-analysis on the effects of antithyroid drugs before RAI treatment suggests that adjunctive antithyroid drugs may reduce biochemical and chemical hyperthyroidism in the weeks after treatment; it may lower cardiac morbidity and mortality (28). Nonetheless, antithyroid drugs may increase the rate of RAI failure: propylthiouracil induces more radioresistance and lower rates of hypothyroidism than methimazole, especially when administered in the week before RAI (28). Accordingly, methimazole should be the preferred option $(27,28)$. The withdrawal of antithyroid drugs could be performed when RAI becomes effective (28). The use of higher doses of RAI can compensate the risk of treatment failure after pretreatment with antithyroid drugs $(27,28)$. A lower mortality after RAI has been reported in two studies in subjects who developed hypothyroidism $(14,15)$. High doses of RAI, which may induce hypothyroidism, could be useful to improve the prognosis of elderly patients avoiding the development of an unrecognized thyroid failure during the follow-up (29).

It has been reported that radioiodine-treated patients may have an increased risk of atrial fibrillation compared with controls (14). Hypercoagulability and hypofibrinolysis may contribute to the increased risk of thromboembolism in hyperthyroid patients with AF. For this reason, anticoagulation should be considered in patients with atrial fibrillation; its beneficial effect should be balanced with the risk of bleeding and other complications of this treatment $(16,27)$. Anticoagulation treatment should be personalized after analyzing the age of the patients, the presence of comorbidities, and the $\mathrm{CV}$ risk factors for stroke.

A careful, long-term follow-up with a strict surveillance for the risk of CVD and fractures should be performed in patients with hyperthyroidism treated with RAI. Drugs to improve bone mineral density should be administered in patients with osteoporosis to rapidly improve the risk of fracture.

Randomized controlled trials are necessary to assess the best treatment to improve $\mathrm{CV}$ mortality in hyperthyroid patients and to evaluate the best approach considering the cause of this dysfunction, the age of the patients, and comorbidities.

\section{CV mortality in SHyper}

Conflicting results have been reported in the large crosssectional and prospective studies assessing the CV morbidity and mortality in patients with endogenous SHyper, reflecting the heterogeneity of the different populations studied (30). Therefore, five meta-analyses have evaluated the CV mortality in patients with SHyper during the last years $(3,5,6,7,8,9)$ (Table 1).

The first meta-analysis performed by Völzke et al. (3) did not report a significant association between SHyper, circulatory mortality, and all-cause mortality. Similarly, the pooled relative risk revealed a nonsignificant difference in CV mortality in SHyper in the metaanalysis by Singh et al. (5), both at baseline and during the follow-up. However, these two meta-analyses only included the small number of studies (respectively two and three studies) available at that time and, therefore, they had a lower power to detect a potential association between SHyper and CV mortality.

The meta-analysis by Ochs published in 2008 included five population-based studies and showed a modestly increased risk for CHD, CV mortality, and total mortality in patients with SHyper (the RR was 1.21 (CI, 0.88 to 1.68 ) for $\mathrm{CHD}, 1.19$ (CI, 0.81 to 1.76 ) for $\mathrm{CV}$ mortality, and 1.12 (CI, 0.89 to 1.42 ) for total mortality (6)). A significantly increased risk of CV disease was found in the general population with SHyper. On the contrary, the risk of $\mathrm{CV}$ and all-cause mortality was particularly increased in individuals with other morbidities. Heterogeneity was observed when pooling all the studies on SHyper and CV mortality, and a lower risk was estimated when pooling higher quality studies (6).

In 2008, another meta-analysis by Haentjens et al. (7) was published. This meta-analysis was performed on seven cohorts, including 290 participants with SHyper. It showed that elderly patients with SHyper and patients with comorbidities had an increased probability of death (7). This risk progressively increased during 10 years after the diagnosis of SHyper: the HR for all-cause mortality was 1.03 (95\% CI, 0.78 to $1.35 ; P=0.83)$ in cohorts from the community and 1.76 (95\% CI, 1.36 to 2.30; $P<0.001)$ in cohorts of patients with comorbidities (7). Some limitations of the epidemiological studies included in these last two meta-analyses $(6,8)$ (one TSH evaluation at baseline and no information on treatment of SHyper during the follow-up) may have affected the results on $\mathrm{CV}$ mortality in the presence of mild thyroid hormone excess.

Recently, more selective criteria have been used in two recent meta-analyses, assessing the CV mortality in patients with SHyper $(8,9)$. Only prospective cohort studies were included, and only cohorts using second- and third-generation TSH assays were selected for the analysis. In fact, the diagnosis of SHyper can be limited using the first-generation TSH assays. Moreover, the authors considered the use of drugs for treatment of SHyper and selected the studies in which the TSH 
evaluation was repeated during the follow-up. Furthermore, these two meta-analyses stratified their analysis according to the degree of TSH suppression.

Yang et al. (8) selected 17 prospective cohort studies in their meta-analysis. They included papers published between 2001 and 2011, which had a duration of follow-up ranging from 2 to 20 years; the majority of these studies were performed in Europe. Only seven of the included studies repeated the TSH evaluation during the follow-up. Moreover, subjects receiving thyroid medications (users of thyroxine and antithyroid drugs) were excluded in ten studies. The results of this metaanalysis showed that SHyper increased the risk of $\mathrm{CV}$ disease in the general population. Moreover, the subgroup analysis showed an increased risk of CV and all-cause mortality in patients with additional comorbidities, confirming previous results $(6,7)$. The overall combined relative risks of CVD for individuals with SHyper compared with the reference group were 1.19 (95\% CI, 1.10 to 1.28$)$ for CVD, 1.52 (95\% CI, 1.08 to 2.13 ) for CV mortality, and 1.25 (95\% CI, 1.00 to 1.55$)$ for all-cause mortality. The omission of each individual study did not significantly change the pooled effects. Moreover, the exclusion of the studies defining SHyper as a suppressed TSH $(<0.1 \mathrm{mU} / \mathrm{l})$ did not significantly influence the results. All the included studies adjusted their results for conventional CV risk factors (age, BMI, blood pressure, diabetes, cholesterol, and smoking), suggesting that SHyper could be an independent risk factor of CVD. No sufficient data were available in these meta-analyses to perform a sub-analysis for the risk of atrial fibrillation and stroke.

Another meta-analysis assessing the individual data from ten prospective cohort studies on the association between SHyper and CV outcome has been recently published in the Archives of Internal Medicine (9). Total mortality and CHD mortality were assessed from all cohorts. CHD event data were analyzed from six cohorts and the data for incident atrial fibrillation events were available from five cohorts. The median follow-up was 8.8 years. Patients receiving thyroid medication or drugs potentially altering thyroid function were excluded. A stratified analysis by age, sex, race, TSH categories, and preexisting CVD was performed.

In age- and sex-adjusted analyses, SHyper was associated with increased total mortality (hazard ratio (HR), $1.24,95 \%$ CI, 1.06 to 1.46 ), CHD mortality (HR, $1.29 ; 95 \% \mathrm{CI}, 1.02$ to 1.62 ), CHD events (HR, 1.21; 95\% CI, 0.99 to 1.46 ), and AF (HR, $1.68 ; 95 \%$ CI, 1.16 to 2.43$)$. When the risk was stratified according to the TSH levels, the results showed that endogenous SHyper was associated with an increased risk of total, CHD mortality, and incident atrial fibrillation, especially in the presence of a TSH level lower than $0.10 \mathrm{mU} / \mathrm{l}$. The risk of stroke did not increase in SHyper.

Therefore, these two recent meta-analyses provide evidence that SHyper may increase CV mortality, particularly in patients with undetectable serum TSH levels. However, still today, the results remain inconclusive and insufficient to recommend treating young patients with SHyper and low-detectable serum TSH levels. In both these recent meta-analyses, all included studies have not provided detailed information on the cause of primary SHyper and, therefore, a sub-analysis for $\mathrm{CV}$ complications related to the etiology of this condition is not available.

The mechanism by which SHyper may be associated with an increased CV risk may be linked to the risk of atrial arrhythmias, especially atrial fibrillation, and the negative changes in $\mathrm{CV}$ function, which have been reported in long-term, untreated, mild thyroid hormone excess $(19,30,31,32,33)$. Few studies have evaluated the effects of medical and RAI treatment to improve CV parameters in SHyper $(34,35,36)$.

Endogenous SHyper is prevalent in areas of iodine deficiency and may be persistent, unrecognized, and untreated mainly in elderly patients. Thus, the evidence of the high CV risk and mortality associated with SHyper suggests that this dysfunction is an important health problem in areas of iodine deficiency and requires guidelines for the best treatment, above all in patients at high CV risk. Further rigorous, randomized controlled studies are necessary to evaluate the effects of the various causes of hyperthyroidism on the clinical outcomes. Randomized, controlled clinical trials are also necessary to assess the benefits of treatment to improve the CV mortality and morbidity of mild thyroid hormone excess.

\section{Declaration of interest}

The author declares that there is no conflict of interest that could be perceived as prejudicing the impartiality of the research reported.

\section{Funding}

This research did not receive any specific grant from any funding agency in the public, commercial or not-for-profit sector.

\section{References}

1 Parry $\mathrm{CH}$. Enlargement of the thyroid gland in connection with enlargement or palpitation of the heart. Collections from the unpublished papers of the late Caleb Hillier Parry 111-125, 1825.

2 Graves RJ. Newly observed affections of the thyroid gland in females. London Medical and Surgical Journal 18357 516-517.

3 Völzke H, Schwahn C, Wallaschofski H \& Dörr M. Review: the association of thyroid dysfunction with all-cause and circulatory mortality: is there a causal relationship? Journal of Clinical Endocrinology and Metabolism 200792 2421-2429. (doi:10.1210/jc.2007-0179)

4 Brandt F, Green A, Hegedüs L \& Brix TH. A critical review and meta-analysis of the association between overt hyperthyroidism and mortality. European Journal of Endocrinology $2011 \mathbf{1 6 5}$ 491-497. (doi:10.1530/EJE-11-0299)

5 Singh S, Duggal J, Molnar J, Maldonado F, Barsano CP \& Arora R. Impact of subclinical thyroid disorders on coronary heart disease, cardiovascular and all-cause mortality: a metaanalysis. International Journal of Cardiology 200828 41-48. (doi:10.1016/j. ijcard.2007.02.027) 
6 Ochs N, Auer R, Bauer DC, Nanchen D, Gussekloo J, Cornuz J \& Rodondi N. Meta-analysis: subclinical thyroid dysfunction and the risk for coronary heart disease and mortality. Annals of Internal Medicine 2008148 832-845.

7 Haentjens P, Van Meerhaeghe A, Poppe K \& Velkeniers B. Subclinical thyroid dysfunction and mortality: an estimate of relative and absolute excess all-cause mortality based on time-to-event data from cohort studies. European Journal of Endocrinology 2008159 329-341. (doi:10.1530/EJE-08-0110)

8 Yang LB, Jiang DQ, Qi WB, Zhang T, Feng YL, Gao L \& Zhao J. Subclinical hyperthyroidism and the risk of cardiovascular events and all-cause mortality: an updated meta-analysis of cohort studies. European Journal of Endocrinology 2012167 75-84. (doi:10.1530/EJE-12-0015)

9 Collet TH, Gussekloo J, Bauer DC, den Elzen WP, Cappola AR, Balmer P, Iervasi G, Asvold BO, Sgarbi JA, Völzke H, Gencer B, Maciel RM, Molinaro S, Bremner A, Luben RN, Maisonneuve P, Cornuz J, Newman AB, Khaw KT, Westendorp RG, Franklyn JA, Vittinghoff E, Walsh JP \& Rodondi N, for the Thyroid Studies Collaboration. Subclinical hyperthyroidism and the risk of coronary heart disease and mortality. Archives of Internal Medicine 2012172 799-809.

10 Goldman MB, Monson RR \& Maloof F. Cancer mortality in women with thyroid disease. Cancer Research 199050 2283-2289.

11 Parle JV, Maisonneuve P, Sheppard MC, Boyle P \& Franklyn JA. Prediction of all-cause and cardiovascular mortality in elderly people from one low serum thyrotropin result: a 10-year cohort study. Lancet $20013 \mathbf{5 8} 861-865$. (doi:10.1016/S0140-6736 (01)06067-6)

12 Gussekloo J, van Exel E, de Craen AJ, Meinders AE, Frolich M \& Westendorp RG. Thyroid status, disability and cognitive function, and survival in old age. Journal of the American Medical Association 2004292 2591-2599. (doi:10.1001/jama.292.21.2591)

13 Osman F, Franklyn J, Holder rL, Sheppard MC \& Gammage MD. Cardiovascular manifestations of hyperthyroidism before and after antithyroid therapy: a matched case-control study. Journal of the American College of Cardiology 200749 71-81. (doi:10.1016/j. jacc.2006.08.042)

14 Metso S, Jaatinen P, Huhtala H, Auvinen A, Oksala H \& Salmi J. Increased cardiovascular and cancer mortality after radioiodine treatment for hyperthyroidism. Journal of Clinical Endocrinology and Metabolism 200792 2190-2196. (doi:10.1210/jc.2006-2321)

15 Franklyn JA, Maisonneuve P, Sheppard MC, Betteridge J \& Boyle P. Mortality after the treatment of hyperthyroidism with radioactive iodine. New England Journal of Medicine 1998338 712-718. (doi:10.1056/NEJM199803123381103)

16 Biondi B \& Kahaly GJ. Cardiovascular involvement in patients with different causes of hyperthyroidism. Nature Reviews. Endocrinology 20106 431-443. (doi:10.1038/nrendo.2010.105)

17 Biondi B. Cardiovascular mortality in subclinical hyperthyroidism an ongoing dilemma. European Journal of Endocrinology 2010162 587-589. (doi:10.1530/EJE-09-1095)

18 Flynn RW, Macdonald TM, Jung RT, Morris AD \& Leese GP. Mortality and vascular outcomes in patients treated for thyroid dysfunction. Journal of Clinical Endocrinology and Metabolism 2006 91 2159-2164. (doi:10.1210/jc.2005-1833)

19 Cappola AR, Fried LP, Arnold AM, Danese MD, Kuller LH, Burke GL, Tracy RP \& Ladenson PW. Thyroid status, cardiovascular risk, and mortality in older adults. Journal of the American Medical Association 2006295 1033-1041. (doi:10.1001/jama. 295.9.1033)

20 Nanchen D, Gussekloo J, Westendorp RG, Stott DJ, Jukema JW. Trompet S, Ford I, Welsh P, Sattar N, Macfarlane PW, Mooijaart SP, Rodondi N \& de Craen AJ, PROSPER. Subclinical thyroid dysfunction and the risk of heart failure in older persons at high cardiovascular risk. Journal of Clinical Endocrinology and Metabolism 201297 852-861. (doi:10.1210/jc.2011-1978)

21 Squizzato A, Gerdes VEA, Brandjes DPM, Büller HR \& Stam J. Thyroid diseases and cerebrovascular disease. Stroke $2005 \mathbf{3 6}$ 2302-2310. (doi:10.1161/01.STR.0000181772.78492.07)
22 Lin HC, Yang LY \& Kang JH. Increased risk of pulmonary embolism among patients with hyperthyroidism: a 5-year follow-up study. Journal of Thrombosis and Haemostasis $2010 \mathbf{8}$ 2176-2181. (doi:10.1111/j.1538-7836.2010.03993.x)

23 Nakazawa HK, Sakurai K. Hamada N, Momotani N \& Ito K. Management of atrial fibrillation in the post-thyrotoxic state. American Journal of Medicine 198272 903-906. (doi:10.1016/ 0002-9343(82)90850-6)

24 Hall P, Lundell G \& Holm LE. Mortality in patients treated for hyperthyroidism with iodine-131. Acta Endocrinologica 1993128 230-234.

25 Nyirenda MJ, Clark DN, Finlayson AR, Read J, Elders A, Bain M, Fox KA \& Toft AD. Thyroid disease and increased cardiovascular risk. Thyroid 200515 718-724. (doi:10.1089/thy.2005.15.718)

26 McDermott MT, Kidd GS, Dodson LE Jr \& Hofeldt FD. Radioiodineinduced thyroid storm. Case report and literature review. American Journal of Medicine $1983 \mathbf{7 5}$ 353-359. (doi:10.1016/00029343(83)91217-2)

27 Bahn RS, Burch HB, Cooper DS, Garber JR, Greenlee MC, Klein I, Laurberg P, McDougall IR, Montori VM, Rivkees SA, Ross DS, Sosa JA, Stan MN \& American Thyroid Association; American Association of Clinical Endocrinologists. Hyperthyroidism and other causes of thyrotoxicosis: management guidelines of the American Thyroid Association and American Association of Clinical Endocrinologists. Thyroid 201121 593-646. (doi:10. 1089/thy.2010.0417)

28 Walter MA, Briel M, Christ-Crain M, Bonnema SJ, Connell J, Cooper DS, Bucher HC, Muller-Brand J \& Muller B. Effects of antithyroid drugs on radioiodine treatment: systematic review and meta-analysis of randomised controlled trials. BMJ $2007 \mathbf{3 3 4}$ 7592-7598. (doi:10.1136/bmj.39114.670150.BE)

29 Franklyn JA, Sheppard MC \& Maisonneuve P. Thyroid function and mortality in patients treated for hyperthyroidism. Journal of the American Medical Association 2005294 71-80. (doi:10.1001/ jama.294.1.71)

30 Biondi B. Should we treat all subjects with subclinical thyroid disease the same way? European Journal of Endocrinology 2008159 343-345. (doi:10.1530/EJE-08-0527)

31 Biondi B, Palmieri EA, Fazio S, Cosco C, Nocera M, Saccà L, Filetti S, Lombardi G \& Perticone F. Endogenous subclinical hyperthyroidism affects quality of life and cardiac morphology and function in young and middle-aged patients. Journal of Clinical Endocrinology and Metabolism 200085 4701-4705. (doi:10. $1210 /$ jc. 85.12 .4701$)$

32 Biondi B \& Cooper DS. The clinical significance of subclinical thyroid dysfunction. Endocrine Reviews 200829 76-131. (doi:10. 1210/er.2006-0043)

33 Biondi B \& Cooper DS. Subclinical thyroid disease. Lancet 2012 379 1142-1154. (doi:10.1016/S0140-6736(11)60276-6)

34 Faber J, Wiinberg N, Schifter S \& Mehlsen J. Haemodynamic changes following treatment of subclinical and overt hyperthyroidism. European Journal of Endocrinology 2001145 391-396. (doi:10.1530/eje.0.1450391)

35 Sgarbi JA, Villaça FG, Garbeline B, Villar HE \& Romaldini JH. The effects of early antithyroid therapy for endogenous subclinical hyperthyroidism in clinical and heart abnormalities. Journal of Clinical Endocrinology and Metabolism 200388 1672-1677. (doi:10.1210/jc.2002-021046)

36 Kaminski G, Michalkiewicz D, Makowski K, Podgajny Z, Szalus N, Ruchala M, Szczepanek E \& Gielerak G. Prospective echocardiographic evaluation of patients with endogenous subclinical hyperthyroidism and after restoring euthyroidism. Clinical Endocrinology 201174 501-507. (doi:10.1111/j.1365-2265. 2010.03957.x)

Received 7 July 2012

Revised version received 13 July 2012

Accepted 16 July 2012 